INDEX TO AUTHORS

353

https://doi.org/10.1017/S0252921100098511 Published online by Cambridge University Press 


\section{INDEX TO AUTHORS}

Ardeberg, A., 109

Arnaud, M., 301

Bahcall, J. N., 326

Beckman, J. E., 185, 67

Bertaux, J. L., 3, 64

Blitz, L., 231

Bloch, J., 222

Bochkarev, N. G., 309

Bowyer, S., 226, 344, 346

Bruhweller, F., 39, 64

Burrows, D., 215

Clark, G., 204

Cowle, L., 287

Cox, D. P., 297

Crivellar1, L., 67

Crovisler, J., 258

Crutcher, R. M., 117

Cugnon, P., 315

Dame, T. M., 235

de Boer, K., 71

Deguch1, S., 274

Dickey, J. M., 258, 268, 269

Edgar, R. J. 297

Fahr, H. J., 28, 32

Feltzinger, J. V., 239

Ferlet, R., 75

Ferrero, R. F., 75

Franco, M. L., 67, 185

Frisch, P. C., 51, 113

Garmire, G. P., 219

Gautier, N., 253

Gerlet, R., 84

Gilden, D. L., 326

Gilra, D. P., 89

Goulet, T., 319

Gry, C., 80, 84

Gwinn, C. R., 281

Hauser, M., 253

Heiles, C., 263, 269

Henry, R. C., 60

Herbstmeier, U., 243

Hobbs, L. M., 189, 280

Holberg, J. B., 91

Jahoda, K., 222, 268

Jakobsen, P., 226

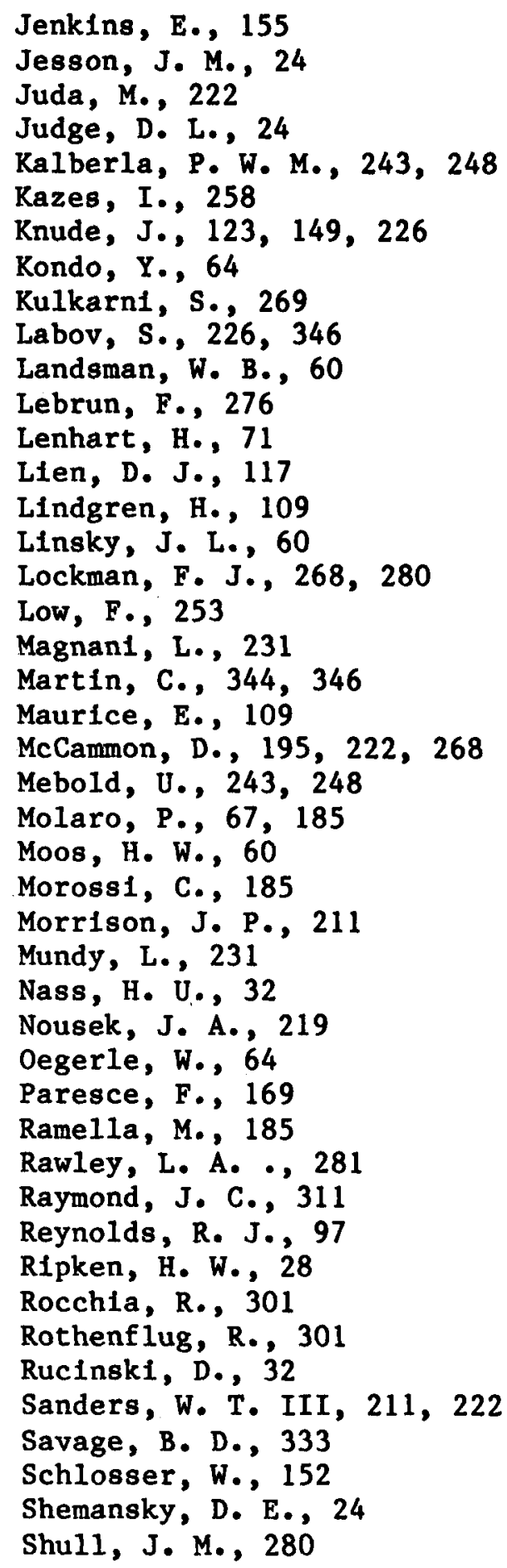


Shuter, W. C. H., 319

Snowden, S., 222

Stencel, R., 64

Stuwe, J., 239

Taylor, J. H., 281

Thaddeus, P., 235

Tinbergen J., 145

van Gorkom, J., 269

Vidal-Madjar, A., 75, 80, 84

Vladilo, G., 67,80

Watson, W., 274

Weaver, G., 219

Weaver, H., 257

Weiler, E., 64

Weisberg, J. M., 281

York, D. G., 51, 80, 113 
The Editors wish to extend special thanks to Teresa L. Evans of the Department of Physics at Catholic University of America for her invaluable assistance in preparing these proceedings. 